\title{
Periodic Merging Networks ${ }^{\star}$
}

\author{
Mirosław Kutyłowski ${ }^{1}$, Krzysztof Loryś ${ }^{2}$, Brigitte Oesterdiekhoff ${ }^{1}$ \\ 1 Heinz Nixdorf Institute and Department of Mathematics \& Computer Science, University of \\ Paderborn, D-33095 Paderborn, Germany, \{mirekk, brigitte\} @uni-paderborn. de, \\ 2 Institute of Computer Science, University of Wrocław, and Dept. of Computer Science, \\ University of Trier, D-54286 Trier, Germany, lorys@ TI. Uni-Trier. DE
}

\begin{abstract}
We consider the problem of merging two sorted sequences on constant degree networks using comparators only. The classical solution to the problem are the networks based on Batcher's Odd-Even Merge and Bitonic Merge running in $\log (2 n)$ time. Due to the obvious $\log n$ lower bound for the runtime, this is timeoptimal.

We present new merging networks that have a novel property of being periodic: for some (small) constant $k$, each processing unit of the network performs the same operations at steps $t$ and $t+k$ (as long as $t+k$ does not exceed the runtime.) The only operations executed are compare-exchange operations, just like in the case of the Batcher's networks. The architecture of the networks is very simple, easy to be laid out. The runtimes achieved are $c \cdot \log n$, for a small constant $c$.
\end{abstract}

\section{Introduction}

Merging is the following problem: given sorted sequences $A$ and $B$ of $n$ keys each, arrange all $2 n$ elements of $A$ and $B$ in one sorted sequence.

Merging is one of the most fundamental problems in computer science and has been intensively studied from theoretical and practical point of view. There have been a lot of efforts to construct optimal merging algorithms in different models. In the parallel setting we have to consider at least two very different situations. For one of them we have a parallel machine performing a non-oblivious computation, for instance a shared memory machine (PRAM). Fascinating sublogarithmic time algorithms have been developed for this model, see for instance [4]. Another possibility is to consider fixed interconnection networks performing oblivious computations. The algorithms developed in this setting might be better suited for implementation, for instance on VLSI circuits. Therefore we are mainly interested in the network model.

\subsection{The Model}

We consider the following architecture: a comparator network algorithm has an underlying graph $G=(V, E)$, where $V$ is the set of processing units (serving as registers

\footnotetext{
* Partially supported by KBN grants $8 \quad$ S503 $002 \quad 07,22$ P301 034 07, DFGSonderforschungsbereich 376 "Massive Parallelität", DFG Leibniz Grant Me872/6-1 and EU ESPRIT Long Term Research Project 20244 (ALCOM-IT); this research was partially done while the second author visited University of Paderborn
} 
as well) and $E$ is the set of the links connecting the processing units. The elements of $E$ will be later called comparators due to the function they perform. We assume that $G$ is a graph of a constant degree. We assume that during execution of the algorithm every processing unit stores exactly one element at each moment. The set $V$ is ordered, the goal of the merging algorithm is to relocate the input elements, so that they form a nondecreasing sequence according to the ordering of $V$. The convention for initial placing two sorted sequences of input elements might be arbitrary as long it is simple.

During a run of the algorithm the elements may be exchanged by neighboring processing units, that is, those processing units that are connected by an comparator in $E$. A comparator $\left(P_{i}, P_{j}\right) \in E$, where $P_{i}<P_{j}$ in the ordering of $V$, acts as follows: the elements held in $P_{i}$ and $P_{j}$ are compared, then the smaller element is placed in $P_{i}$ and the bigger one is placed in $P_{j}$. The processing unit $P_{i}\left(P_{j}\right)$ is said to be on the minimum side (on the maximum side) of the comparator $\left(P_{i}, P_{j}\right)$.

A comparator network algorithm consists of parallel compare-exchange steps. A parallel compare-exchange step uses a matching in the graph $G$, that is a set of edges $E^{\prime} \subseteq E$, such that no element of $V$ belongs to two edges in $E^{\prime}$. During the parallel step all comparators from $E^{\prime}$ act in parallel.

We say that a comparator network algorithm is periodic with period $k$ if for every moment $t$ of the computation, the parallel compare-exchange steps $t$ and $t+k$ use the same matching, that is, the same groups of comparators. The steps $(i-1) \cdot k+1$ through $i \cdot k$ are called the $i$ th round of the algorithm.

Comparator network algorithms are often described in a different way. The processing units are replaced by wires. Then step $t$ of a compare-exchange algorithm corresponds to level $t$ of comparators in the model with wires. If the algorithm is not periodic, such an interpretation is necessary in order to implement the algorithm on a network of a fixed degree.

\subsection{Previous Work}

Merging by comparator networks has been studied for a long time. The most famous merging networks are the Odd-Even Merger and the Bitonic Merger of Batcher [1], and the Balanced Merger of Dowd et al. [5]. They run in time $\log (2 n)$ and use $n \cdot \log n+$ $O(n)$ comparators. There has been a lot of research on these networks and they have been modified in many ways (see [13], [11], [3], [2], [10]). Milterson et al. [9] prove that the minimal number of comparators needed for merging two $n$-element sequences is at least $n \cdot \log n-O(n)$, improving the previous results (see Knuth [7]). It follows that the networks by Batcher are asymptotically optimal. This result also shows that merging two $n$-element sequences on a comparator network requires $\log n-O(1)$ time.

Recently, periodic comparator network algorithms for sorting have been intensively studied. After a number of algorithms with a non-constant period ([5], [2], [10] and [3]) finally some fast periodic sorting networks of constant period have been found:[12], [6] and [8]. The main result of the last paper mentioned is the so called "periodification scheme". It says that any sorting comparator network algorithm may be rebuilt so that we obtain a constant period algorithm. This modification causes a slowdown of factor $\log n$ in the case of fast algorithms. 
In a certain sense we expand the ideas of [8]. However, the periodification scheme cannot yield a time-optimal merging algorithm due to the slowdown inherent in the periodification scheme. Therefore we develop a direct solution.

\subsection{The New Results}

Theorem 1. There is a periodic comparator network of period 4 that merges two sorted sequences of $n$ items in time $10 \cdot \log _{3} n \approx 6.3 \cdot \log n$.

It is not difficult to find an example showing that $10 \cdot \log _{3} n-O(1)$ is really the worst case runtime of our algorithm. In a forthcoming journal version of this paper we present further variants of Theorem 1, where we reduce the period to 3 or reduce the runtime to about $2.84 \log n$. (However, they require a more tedious analysis.) The last result differs only by a small constant factor from the lower bound for merging in comparator networks, although the restrictions imposed on our network are very strong.

\section{Construction of the Network}

In this section we describe the techniques used by our algorithm. We explain the underlying ideas before we go into (rather technical) details.

It is well known that comparator networks that sort all inputs consisting of 0 's and 1's sort correctly arbitrary input sequences (so called 0-1-Principle [7]). A similar phenomenon holds for merging (and can be proved in an analogous way):

Proposition 1. If a comparator network algorithm merges two sorted sequences consisting solely of 0 's and 1 's, then it correctly merges any two sorted input sequences.

By Proposition 1, for the rest of the paper we may consider only the input sequences consisting of 0's and 1's.

Our merging algorithm uses a network $M$ the processing units of which are arranged in a $p \times q$-rectangle, where $p=3^{q}, q$ is even. Let $P_{i, j}$ denote the processing unit of $M$ in the row $i$ and the column $j$, for $i \in\{1,2, \ldots, p\}$ and $j \in\{1,2, \ldots, q\}$. Let $C_{j}=\left\{P_{i, j} \mid i \leq p\right\}$ denote the $j$ th column of $M$. We order the nodes of $M$ by the snake-like ordering: $P_{i, j} \prec P_{i^{\prime}, j^{\prime}}$ if and only if

$$
\left(i>i^{\prime}\right) \quad \vee \quad\left(i=i^{\prime} \wedge j<j^{\prime} \wedge i \text { odd }\right) \quad \vee \quad\left(i=i^{\prime} \wedge j>j^{\prime} \wedge i \text { even }\right)
$$

The algorithm has a strange property that at each step of the computation if an element moves from column $C_{i}$ to $C_{j}$, then every element stored in $C_{i}$ moves to $C_{j}$. (This property holds only for the input sequences consisting of 0 's and 1's, and under the assumption that the comparators may switch equal elements. Thereby our analysis collapses if we do not use the 01-Principle. This is an interesting example how powerful this principle is.) So we may interpret that the algorithm moves the columns around the network. The purpose of these movements is that at different positions different sets of comparators are applied inside a column. The input allocation to this network has the property that the number of 1's at each column is the same up to one. Since the columns move separately, this property will be preserved all the time. After sorting each column, the contents of the network is sorted except of at most one row. Some additional rounds suffice to sort this row. 


\subsection{Input Allocation.}

Definition 1. We say that a column $C_{i}$ is of the form $1^{d}(01)^{e} 0^{*}$ if $d$ bottom positions in $C_{i}$ are occupied by 1 's, the $p-2 e-d$ top positions in $C_{i}$ are occupied by 0 's, and in the remaining 2 e positions there are interchangeably 0 's and 1's, starting with a 0 at position $d+1$.

It is crucial that the input allocation fulfills the following property:

Property 1. After loading the input sequences

- the number of 1 's in the columns of $M$ may differ by at most 1 ,

- each column has the form $1^{d}(01)^{e} 0^{*}$ for some $e$ and $d$.

Property 1 is obviously fulfilled for instance for the following easy scheme: The elements of $A$ are loaded into the odd rows, and the elements of $B$ into the even rows; elements of $A(B)$ are placed according to the snake-like ordering.

Our algorithm will be designed in such a way that Property 1 will hold during the whole computation.

\subsection{Horizontal Steps}

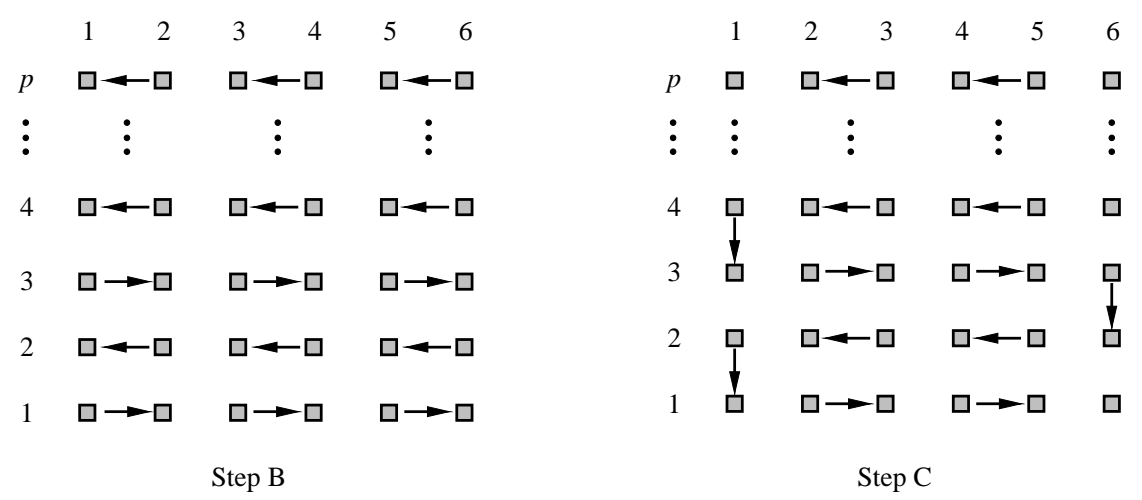

Fig. 1. The parallel Steps B and C

The algorithm of Theorem 1 executes periodically 4 different steps, called A, B, C, and D. The Steps A and D, called vertical, affect each column separately. The Steps $\mathrm{B}$ and $\mathrm{C}$, called horizontal, exchange elements between consecutive columns in the same row (see Fig. 1). (An exception are the comparators in the leftmost and rightmost columns at Step C.) More precisely, Step B uses comparators $\left(P_{i, j}, P_{i, j^{\prime}}\right), i \leq p, j \leq q$, where

$$
j^{\prime}=j+1 \quad \text { if } i, j \text { are odd, } \quad j^{\prime}=j-1 \quad \text { if } i, j \text { are even. }
$$


Step C uses comparators $\left(P_{i, j}, P_{i, j^{\prime}}\right)$, where

$$
j^{\prime}=j+1, \quad \text { if } i \text { is odd, } j \text { is even, } j<q \quad j^{\prime}=j-1, \quad \text { if } i \text { is even, } j \text { is odd. }
$$

Additionally, at $C_{1}$ there are comparators $\left(P_{i, 1}, P_{i-1,1}\right)$ for each even $i \leq p$, and at $C_{q}$ there are comparators $\left(P_{i, q}, P_{i-1, q}\right)$ for each odd $i \leq p, i \neq 1$.

Definition 2. A column with the contents of the form $1^{d}(01)^{e} 0^{*}$ is classified as an Ecolumn if $d$ is even, and $e>0$, or as an $O$-column if $d$ is odd and $e>0$. If $e=0$, then the column is said to be an $S$-column. ("S" stands for "sorted", "O" for "odd", and "E” for "even".)

The lowest $d$ positions in this column form the foot of the column, the next $2 e$ positions form the 01-region.

We say that a column is an E/S-column if it is an E-or S-column, and we call it an $\mathrm{O} / \mathrm{S}$-column if it is an $\mathrm{O}$ - or $\mathrm{S}$-column.

In the following lemma we show that under certain circumstances the columns exchange their contents during horizontal steps. This is a key to our construction.

Lemma 1. Consider the columns $C_{i}$ and $C_{i+1}$ of $M$ and assume that $C_{i}$ is an O/Scolumn, $C_{i+1}$ is an E/S-column and that the number of 1 's in them differ by at most 1 . Assume further that during Step B or C comparators connect the columns $C_{i}$ and $C_{i+1}$. Then during this step:

(a) $C_{i}$ and $C_{i+1}$ exchange their items provided that at least one of them is not an $S$ column.

(b) $C_{i}$ and $C_{i+1}$ sort together according to the snake-like ordering of $M$ provided that they are both $S$-columns.

Proof. Point (b) is obvious. For (a) assume that $C_{i}$ is an O-column and $C_{i+1}$ is an Ecolumn. Let $C_{i}$ be of the form $1^{d}(01)^{e} 0^{*}$ and $C_{i+1}$ be of the form $1^{d^{\prime}}(01)^{e^{\prime}} 0^{*}$. Suppose that $d<d^{\prime}$. Then the $d$ bottom positions in $C_{i}$ and $C_{i+1}$ are occupied by 1 's and this does not change at the horizontal step. So we may say that within the first $d$ rows $C_{i}$ and $C_{i+1}$ exchange their contents. In the next $2 e$ rows the 1's of $C_{i}$ are at the minimum sides of the comparators, whereas the 0 's are at the maximum sides of the comparators. Thus the comparators move these 0 's and 1's to column $C_{i+1}$ no matter what are the contents of $C_{i+1}$ (equal items are supposed to be switched.) Now let us consider the positions above the row $d+2 e$ in both columns. $C_{i}$ contains no 1 's there, so we have to show that all 1's from $C_{i+1}$ in this part are moved to $C_{i}$ at the horizontal step. Column $C_{i}$ contains $d+e$ ones, hence $C_{i+1}$ contains at most $d+e+1$ ones. Assume that $C_{i+1}$ contains at least one 1 above the row $d+2 e$. Since $d$ ones are contained in the first $d$ rows, the next $2 e$ rows contain at least $e$ ones, there is exactly one 1 above the row $d+2 e$ in $C_{i+1}$. Since $C_{i+1}$ is an E-column, its last one is at an even row and therefore must be moved into $C_{i}$ during this step. So we see that also above the row $d+2 e$ the columns $C_{i}$ and $C_{i+1}$ exchange their 1's.

The other cases can be checked in a similar way. 


\subsection{Step A and Movements of the Columns}

Lemma 1 shows that O- and E-columns exchange their contents at Steps B and C. For our eye observing the computation it looks like switching positions by the columns. So for a computation consisting of Steps B and C, only, we would see only the columns moving around the network. We shall call them moving columns. Our algorithm performs also vertical Steps D and A. They influence each of the moving columns separately. How the vertical steps sort the moving columns is an important issue, but we require that immediately before Step B the odd column are O/S-columns, and the even columns are E/S-columns. (Otherwise we could not use Lemma 1.) For this purpose, Step A applies the comparators $\left(P_{i, j}, P_{i-1, j}\right)$, where $i, j$ are of different parity (see Fig. 2). By the definition we get immediately the following property:

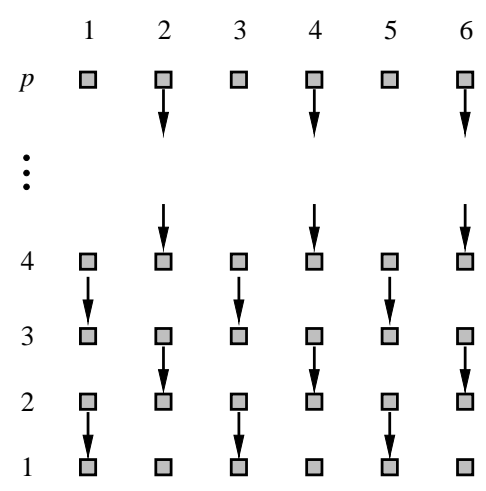

Fig. 2. The parallel Step A

Lemma 2. (a) Suppose that each column is an E-, O- or $S$-column. Then by performing Step A, each odd column of $M$ becomes an O/S-column, each even column becomes an E/S-column.

(b) At Step $C$ the leftmost column becomes an $O / S$-column and the rightmost column becomes an E/S-column.

By Lemma 2 we see that immediately before Step B the O- and E-columns are in the right positions in order to apply Lemma 1 . So

immediately before Step C each odd column is an E/S-column and each even column is an $\mathrm{O} / \mathrm{S}$-column.

Hence again Lemma 1 can be applied and together with Lemma 2(b) we get:

immediately before Step D each odd column is an O/S-column and each even column is an E/S-column.

By the above observations it is easy to see that the sequence of the steps has been arranged in such a way that:

(i) During Steps B and C the E-columns move to the left, the O-columns move to the right. The only exception are the leftmost and the rightmost columns at Step $\mathrm{C}-$ they 
cannot move any further. Instead they change their status: the leftmost column becomes an O/S-column and the rightmost column becomes an E/S-column.

(ii) During Step D some changes within unsorted columns are made. For this reason an E-column (O-column) may loose its status of an E-column (O-column), but at the next Step A it recovers the proper status.

(iii) Every column that is not sorted moves back and forth through $M$, the changes of the direction of the movement occur only at the moment when it reaches the rightmost or the leftmost column. An immediate consequence is that we sort the elements of each column separately.

\subsection{Construction of Step D}

Now we describe Step D responsible for sorting the columns. In a column $C_{i}$ it uses all comparators $\left(P_{j, i}, P_{j-3^{w(i)}, i}\right)$, for $j>3^{w(i)}$ having the same parity as $i$. The choice of the parameter $w(i)$ will be discussed in the next section. The comparators $\left(P_{i, j}, P_{i, j-3^{w(i)}}\right)$ will be called jump comparators and $3^{w(i)}$ the size of the jump.

Note that all jump comparators within a column have the same length and either originate in odd rows and point to even rows, or originate in even rows and point to odd rows. Thereby, the comparators have no common endpoints and the definition is sound. For the inputs considered, immediately before Step D odd columns are O/Scolumns and even columns are E/S-columns. Thus in the odd (even) columns the jump comparators originate at odd (even) rows where we may expect to find 1's of the 01region. The idea is that with some luck some of these 1's jump into the places occupied previously by 0's of the 01-region and thereby reduce the size of the 01-region. Of course, the effect depends on the relative size of the 01-region and the jump size:

Lemma 3. Suppose that immediately before Step D a column $C_{i}$ has the form $1^{d}(01)^{e} 0^{*}$, where $d$ has the same parity as $i$. Let $\ell$ be the jump size at column $C_{i}$. Then after Step D:

(a) For $e<\ell / 2$, the contents of $C_{i}$ remains unchanged.

(b) For $\ell / 2 \leq e<\ell$, column $C_{i}$ gets the form $1^{d+2 e-\ell+1}(01)^{\ell-1-e} 0^{*}$.

(c) For $e \geq \ell$, column $C_{i}$ gets the form $1^{d+\ell}(01)^{e-\ell} 0^{*}$.

Proof. Of course, performing Step D leaves the old foot intact, and no 1 can move above the 01-region. So the only part that may change is the 01-region.

Case $(a)$ : If $\ell>2 e$, then each jump comparator has either the maximum side in the foot, or the minimum side above the 01-region. Such a comparator does not change the contents of its endpoints, hence performing Step D has no affect.

Case (b) (see Fig. 3): As already seen, the jump comparators originating between rows $d+1$ and $d+\ell$ compare two 1's and thereby do not change the contents of the column. The jump comparators with the minimum side at rows $d+\ell+1$ up to $d+2 e$ have 1's on the minimum side and 0's on the maximum side. Therefore their 1's jump down. There are $e-(\ell-1) / 2$ such comparators, so these ones move into rows $d+1, d+3, \ldots, d+$ $2 e-\ell$. Since $2 e-\ell<\ell$, all these 1 's are moved into the part of the 01 -region inside which the ones remain on their places. Thereby, the foot grows up to row $d+2 e-\ell+1$, 


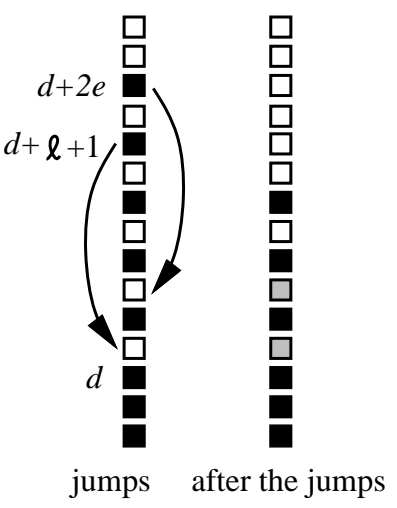

Case (b)

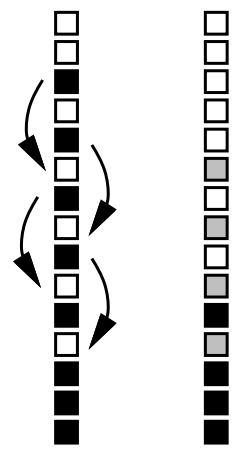

jumps after the jumps

Case (c)

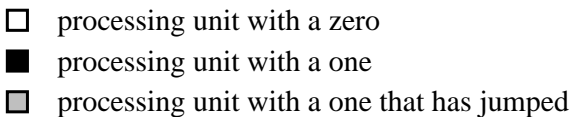

Fig. 3. Cases (b) and (c) of Lemma 3

and there is a new 01-region containing the remaining $\ell-1-e$ ones that have not been moved. So the column gets the form $1^{d+2 e-\ell+1}(01)^{\ell-1-e} 0^{*}$.

Case (b) (see Fig. 3): The difference to the previous case is that not all 1's that jump down fit into the $\ell$ lowest rows of the old 01-region - there are too many jumping 1's. The 1's from the 01-region that remain on their places and the 1's jumping into the lowest $\ell$ rows of the old 01-region cause the foot to grow by $\ell$. Above the row $d+\ell$ there are only 1 's that have been moved, they form a new 01-region with $e-\ell$ ones. So the column gets the form $1^{d+\ell}(01)^{e-\ell} 0^{*}$.

\subsection{Jump Sizes at Step D}

We have still to determine the sizes of the jumps so that the columns are sorted fast. In order to sort a column it suffices to reduce gradually the size of the 01-region at Step D. The size of the jumps is essential: if they are too long, then the size is not reduced (see Lemma 3(a)); if they are too short, then the decrease of the size is not substantial (see Lemma 3(c)). A lucky situation is when the size of the 01 -region is $2 e=3 \ell-1$, where $\ell$ is size of the jumps at this column. Then by Lemma 3(c), at step D the size of the 01-region is reduced to $2(e-\ell)=\ell-1$, that is, to about one third of the original size. Thus in at $\operatorname{most} \log _{3} p$ rounds we could reduce the size of a 01 -region to 0 .

These ideas can be implemented on a periodic network as follows: For a given column at some initial position we first let it move to $C_{1}$. Eventually every moving column reaches $C_{1}$ (see Subsection 2.3). In the meantime, the Steps D and A may 
reduce the size of the 01-region of this column, but we do not rely on this improvement. Once a moving column arrives at $C_{1}$ the real game starts.

Between consecutive Steps D two horizontal moves are executed, so a moving column will be at the following positions while executing Step D:

$C_{1}, C_{3}, C_{5}, \ldots, C_{q-1}, C_{q}, C_{q-2}, \ldots, C_{2} \ldots$. Note that immediately before Step D at $C_{1}, C_{3}, C_{5}, \ldots, C_{q-1}$ this moving column will be an O/S-column, and at $C_{q}, C_{q-2}, \ldots, C_{2}$ this column column will be an E/S-column. So we define the jumps of Step D as follows:

- at odd (even) columns the jump comparators of Step D originate at odd (even) rows,

- at columns $C_{1}, C_{3}, C_{5}, \ldots, C_{q-1}$ the sizes of the jumps are equal, respectively, to $p / 3, p / 9, \ldots, p / 3^{q / 2}$,

- at columns $C_{q}, C_{q-2}, \ldots, C_{2}$ the sizes of the jumps are equal, respectively, to $p / 3^{q / 2+1}, p / 3^{q / 2+2}, \ldots, 1$.

The points of origin of the jump comparators (in even or odd rows) are chosen so that Lemma 3 can be applied, and a moving column starting its movement at $C_{1}$ will be affected by jumps of size $p / 3, p / 9, \ldots, 1$, as expected.

\section{Runtime Analysis}

Conceptually the computation consists of two phases: during the first phase all columns become sorted, but may be they are still in a wrong order causing one row to be unsorted. During the second phase the order of the columns is corrected.

Lemma 4. Assume that at a given column the comparators of Step D have length $\ell$ and the 01-region has size $2 e$ so that $2 e \leq 3 \ell-1$. Then Step $D$ reduces the size of the 01 -region at this column to at most $\ell-1$.

Proof. Let $x$ denote the size of the 01-region after Step D. We apply Lemma 3 in three separate cases: If $e<\ell / 2$, then $x=2 e<\ell$ by Lemma 3(a). If $\ell / 2 \leq e<\ell$, then by Lemma 3(b), $x=2(\ell-1-e) \leq \ell-2$. For $e \geq \ell$ but satisfying $2 e \leq 3 \ell-1$, by Lemma 3(c) we get $x=2(e-\ell) \leq \ell-1$.

Corollary 1. Consider a moving column immediately $i$ rounds after the moment it has reached $C_{1}$ before Step $D$ for the first time. Then its 01 -region has size at most $p / 3^{i}-1$.

Proof. The proof is by induction on $i$. For $i=0$, it is obvious, since a 01-region may not be bigger than $p-1$, because $p$ is odd and the size of the 01-region is even. Assume that the claimed property holds for $i$ and consider the next round. Step A may only decrease the size of the 01-region. Performing Steps B and C moves the column to the next position where Step D performs jumps of length $p / 3^{i+1}$. By Lemma 4 , Step D reduces the size of the 01 -region in this column to at most $p / 3^{i+1}-1$.

By Corollary 1 , a moving column that arrives back at $C_{1}$ has a 01 -region of size at most $p / 3^{q}-1=0$. Thus the column is sorted. Let us count how much time elapses until this moment:

(i) In the worst case, in order to reach $C_{1}$ immediately before Step $\mathrm{D}$ the column needs to move to the right border, change its direction (at Step C), and move back to the 
left border. Together, it takes at most $2 q$ horizontal steps, that is, at most $4 q$ steps.

(ii) After arriving at $C_{1}$ the moving column has to get to the right border and then back to the left border in order to become sorted. It requires $2 q$ horizontal steps, that is, $4 q$ steps.

We conclude that during the first $8 q$ steps every column becomes sorted.

By Lemma 3, once all columns are sorted we only reorder the columns. Steps A and D have no affect anymore, Steps B and C mimic Odd-Even Transposition Sort of $q$ items. Since $q$ steps of Odd-Even Transposition Sort suffice to sort $q$ items [7], relocating the sorted columns requires at most $q$ horizontal steps, that is, $2 q$ steps of our algorithm.

Concluding, the algorithm needs together at most $10 q$ steps. Since $n=p \cdot q / 2=$ $3^{q} \cdot q / 2$, we have $q \leq \log _{3} n$ and therefore the runtime of the algorithm is bounded by $10 \cdot \log _{3} n$ completing the proof of Theorem 1 .

\section{Acknowledgment}

Some elements of our construction (jump comparators) have been considered earlier by Grzegorz Stachowiak in the context of sorting networks. The second author would like to thank F. Meyer auf der Heide for inviting him to Paderborn, where this research has started.

\section{References}

1. Kenneth E. Batcher. Sorting networks and their applications. In AFIPS Conf. Proc. 32, pages 307-314, 1968.

2. Ronald I. Becker, David Nassimi, and Yehoshua Perl. The new class of g-chain periodic sorters. In Proc. 5th ACM-SPAA, pages 356-364, 1993.

3. Gianfranco Bilardi. Merging and sorting networks with the topology of the Omega network. IEEE Transactions on Computers, 38:1396-1403, 1989.

4. Allan Borodin and John E. Hopcroft. Routing, merging, and sorting on parallel models of computation. J. Comput. Syst. Sci., 30(1):130-145, 1985.

5. Martin Dowd, Yehoshua Perl, Larry Rudolph, and Michael Saks. The periodic balanced sorting network. Journal of the ACM, 36:738-757, 1989.

6. Marcin Kik, Mirosław Kutyłowski, and Grzegorz Stachowiak. Periodic constant depth sorting networks. In Proc. 11th STACS, pages 201-212, 1994.

7. Donald E. Knuth. The Art of Computer Programming, Volume 3: Sorting and Searching. Addison-Wesley, Reading, MA, 1973.

8. Mirosław Kutyłowski, Krzysztof Loryś, Brigitte Oesterdiekhoff, and Rolf Wanka. Fast and feasible periodic sorting networks of constant depth. In Proc. 35th IEEE-FOCS, pages 369380, 1994.

9. Peter Bro Miltersen, Mike Paterson, and Jun Tarui. The asymptotic complexity of merging networks. In Proc. 33rd IEEE-FOCS, pages 236-246, 1992.

10. David Nassimi, Yehoshua Perl, and Ronald I. Becker. The generalized class of g-chain periodic sorting networks. In Proc. 8th IPPS, pages 424-432, 1994.

11. Yehoshua Perl. Better understanding of Batcher's merging networks. Discrete Applied Mathematics, 25:257-271, 1989. 
12. Uwe Schwiegelshohn. A shortperiodic two-dimensional systolic sorting algorithm. In International Conference on Systolic Arrays, pages 257-264, 1988.

13. Robert Sedgewick. Data movement in odd-even merging. SIAM J. Comput., 7:239-272, 1978. 